\title{
A Multivalent Vaccine Containing Actinobacillus pleuropneumoniae and Mycoplasma hyopneumoniae Antigens Elicits Strong Immune Responses and Promising Protection in Pigs
}

\author{
Hoai Thu Dao ${ }^{1,5}(\mathbb{D})$, Woo-Sung Shin ${ }^{2}(\mathbb{D})$, Van Tan Do $^{1,5}\left(\mathbb{D}\right.$, Quang Lam Truong ${ }^{3}$, \\ Jong-Young Choi ${ }^{1,4}$ (D) and Tae-Wook Hahn ${ }^{1,2}$
}

${ }^{1}$ College of Veterinary Medicine and Institute of Veterinary Science, Kangwon National University, Chuncheon 24341, Korea.

${ }^{2}$ Innovac Co., Kangwon National University, 1 Kangwondaehak-gil, Chuncheon 24341, Korea.

${ }^{3}$ Key Laboratory of Veterinary Biotechnology, Faculty of Veterinary Medicine, Vietnam National University of Agriculture, Hanoi, Vietnam.

${ }^{4}$ Dodam Veterinary Clinics, Seoul 04716, Korea.

${ }^{5}$ Institute of Veterinary Research and Development of Central Vietnam, Vietnam.

\begin{abstract}
Actinobacillus pleuropneumoniae (App) and Mycoplasma hyopneumoniae (Mhp) cause porcine pleuropneumonia and mycoplasmal pneumonia, respectively, and have serious impacts on the swine industry because they retard the growth of pigs. To protect pigs against these diseases, we have developed a multivalent vaccine consisting of App bacterins, APP RTX toxins (Apx toxins), and Mhp bacterin and adhesin protein. This vaccine induced the production of higher levels of antibodies against App and Mhp than the commercial vaccine (Nisseiken Swine APM Inactivated Vaccine). Furthermore, the vaccine efficiently protected pigs against virulent App challenge, showing promise as an efficient vaccine for the prevention of two important respiratory diseases, porcine pleuropneumonia and mycoplasmal pneumonia.
\end{abstract}

Keywords: Actinobacillus pleuropneumoniae, Mycoplasma hyopneumoniae, multivalent vaccine, immune responses, protection efficacy

*Correspondence: twhahn@kangwon.ac.kr; +82-33-2508671

(Received: May 29, 2020; accepted: January 04, 2021)

Citation: Dao HT, Shin W-S, Do VT, Truong QL, Choi J-Y, Hahn T-W. A Multivalent Vaccine Containing Actinobacillus pleuropneumoniae and Mycoplasma hyopneumoniae Antigens Elicits Strong Immune Responses and Promising Protection in Pigs. J Pure Appl Microbiol. 2021;15(1):164-174. doi:10.22207/JPAM.15.1.11

(C) The Author(s) 2021. Open Access. This article is distributed under the terms of the Creative Commons Attribution 4.0 International License which permits unrestricted use, sharing, distribution, and reproduction in any medium, provided you give appropriate credit to the original author(s) and the source, provide a link to the Creative Commons license, and indicate if changes were made. 


\section{INTRODUCTION}

Actinobacillus pleuropneumoniae (App) is the causative agent of porcine pleuropneumonia, which affects the swine industry worldwide ${ }^{1}$. Vaccination is one of the most promising ways to control and prevent porcine pleuropneumonia ${ }^{2,3}$. To date, the most commonly used commercial vaccines are inactivated vaccines (Ingelvac $\mathrm{H}$, Suvaxyn Respifend APP, Aptovac, Neumosuin, etc.) and subunit vaccines (Porcilis AP, AP125RX, etc.) because of their advantages ${ }^{1}$. First, inactivated vaccines are safer than live-attenuated vaccines because they cannot revert to virulence and reduce the risk of recipients becoming carriers ${ }^{4,5}$. Second, they can be combined with antigens from other pathogens to form multivalent vaccines to prevent infection with several pathogens. Third, they are ecologically safe because they do not introduce live pathogens or live recombinant organisms to the environment ${ }^{6,7}$.

Eighteen serotypes of App have been identified ${ }^{8}$, which has contributed to the difficulty of effective control and prevention of the disease. Most App serotypes secrete one or more Apx toxins, which are closely related to the virulence of each serotype 9 . Four kinds of Apx exotoxins with different functions and virulence have been identified. Of these, ApxI, II, and III, which are considered the most important virulence factors, can be produced and secreted into culture supernatants, while ApxIV, an in vivo expressed protein, can be detected during natural infection ${ }^{1}$. Numerous commercial and experimental vaccines using Apx toxins have been developed because of their high immunogenicity. App serotypes 1 and 5 , the most virulent serotypes that produce both ApxI and ApxIl toxins ${ }^{10,11}$, are the predominant serotypes in Korea ${ }^{12}$. The less virulent App serotype 2, which produces ApxII and ApxIII toxins, also contributes to infection in pigs in Korea ${ }^{12}$. These serotypes have been used widely in the development of App bacterin and Apx toxoid vaccines. In this study, we developed a multivalent vaccine containing App serotype 1, 2, and 5 bacterins and ApxI, II, and III toxoids to protect pigs against virulent App serotype 1, 2, and 5 infections.

Mycoplasma hyopneumoniae (Mhp) is the causative agent of porcine enzootic pneumonia (mycoplasmal pneumonia) ${ }^{13}$, and has huge economic impacts in the swine industry ${ }^{14}$. Vaccination can effectively reduce its clinical signs and the severity of lung lesions, and is therefore widely used to prevent Mhp infection ${ }^{13,15}$. The current commercial vaccines against $\mathrm{Mhp}$ include bacterin vaccines, subunit vaccines, and combination vaccines ${ }^{13,14}$. Although these vaccines can reduce the severity of Mhp infection, vaccines that can confer better protective efficacy and reduce transmission are still required.

P97 protein is a surface adhesin that is highly conserved among strains of $\mathrm{Mhp}^{16,17}$. P97 is well characterized and its fragments have been expressed in Escherichia coli (E. coli) and evaluated as candidate vaccine components ${ }^{13}$. Recombinant repeat regions of $\mathrm{P} 97$ conferred good protection and represented a promising candidate for developing Mhp vaccines ${ }^{18-20}$.

Coinfection with App and Mhp exacerbates the severity of these respiratory diseases in pigs ${ }^{21,22}$, and results in more severe lung lesions and poorer growth. Furthermore, coinfection or sequential infection with App and Mhp is commonly detected in the field. Therefore, the control of App and Mhp coinfection is important for reducing economic losses.

In this study, we produced a multicomponent vaccine containing App serotype 1, 2, 5 bacterins, Apx toxoids, inactivated Mhp, and recombinant P97 (rP97) from Mhp with the aim of reducing injection-induced stress in pigs. The immunogenicity and protective efficacy of the vaccine against virulent App challenge were evaluated and compared with those of a commercial vaccine. The results suggest that this vaccine is a promising candidate to protect pigs against App and Mhp.

\section{MATERIALS AND METHODS}

\section{Strains, growth conditions, and primers}

App serotypes 1, 2, and 5 were isolated from the lungs of infected pigs in Korea and are available in the Laboratory of Infectious Diseases, Kangwon National University. App strains were grown in mycoplasma broth base (PPLO; Oxoid Ltd., UK) supplemented with $10 \mu \mathrm{g} / \mathrm{mL}$ of nicotinamide adenine dinucleotide (NAD; Merck, Germany) at $37^{\circ} \mathrm{C}$ and $5 \% \mathrm{CO}_{2}$ atmosphere. The primers used to identify App are listed in Table 1. 
Mhp was isolated from a sample from an infected pig and was cultured in modified Friis medium ${ }^{23}$ at $5 \% \mathrm{CO}_{2}$ and $37^{\circ} \mathrm{C}$.

\section{Preparation of inactivated App}

Whole-cell, formalin-inactivated bacterins were prepared from App serotypes 1, 2, and 5 as described previously ${ }^{24}$. Briefly, bacteria were grown to reach the mid-exponential phase in PPLO containing $10 \mu \mathrm{g} / \mathrm{mL} \mathrm{NAD}$ at $37^{\circ} \mathrm{C}$. The bacteria were harvested by centrifugation ( $8000 \mathrm{x} \mathrm{g}$ for 20 min at $4^{\circ} \mathrm{C}$ ) and the pellets were washed twice with phosphate-buffered saline (PBS). Formalin was added at $2 \%$ of the total volume and the mixtures were incubated for $4 \mathrm{~h}$ at $37^{\circ} \mathrm{C}$ with shaking at 180 $\mathrm{rpm}$. After three washes with PBS, the inactivated bacteria were resuspended in PBS, subcultured to verify successful inactivation, and then frozen at $-70^{\circ} \mathrm{C}$ until use.

\section{Production of Apx toxoids}

Apx I, Apx II, and ApxIII were produced, optimized, and purified from App serotypes 1, 2 , and 5 as described in our previous study ${ }^{25}$. The purified Apx toxins were inactivated by $0.4 \%$ formalin ${ }^{26}$ overnight at $4^{\circ} \mathrm{C}$ and the formalin was removed by ultrafiltration using Amicon Ultra-15 centrifugal filters with a 10-kDa molecular weight cutoff (Merck Millipore Ltd., Ireland). The collected toxoids were stored at $-70^{\circ} \mathrm{C}$ until use. The purity and the concentration of the toxoids were estimated using SDS-PAGE as previously described $^{25}$.

\section{Preparation of inactivated Mhp}

The Mhp vaccine strain HID3140 was cultured in modified Friis medium ${ }^{23}$ for 10 days at $37^{\circ} \mathrm{C}$ and $5 \% \mathrm{CO}_{2}$ until it reached $5 \times 10^{8}$ colorchanging units (CCU). Mhp was inactivated as described by Matthijs et al. ${ }^{23}$. Briefly, binary ethyleneimine (BEI) was added to the liquid culture and incubated at $37^{\circ} \mathrm{C}$ for $24 \mathrm{~h}$ with shaking set to $100 \mathrm{rpm}$. Then, BEI was neutralized by adding sodium thiosulfate (Sigma Aldrich, USA) and cultures were incubated for $24 \mathrm{~h}$ with shaking (100 rpm). Subcultures of the inactivated Mhp were incubated for one week to confirm the inactivation.

\section{Construction and expression of rP97 in E. coli}

The plasmid pET-DEST42 containing the fusion gene LTB and R1R2 of P97 was previously constructed in our lab ${ }^{18}$ and transformed into E. coli BL21. The rP97 was expressed and purified from E. coli lysate using HisPur Ni-NTA affinity chromatography (Thermo Fisher Scientific, USA) ${ }^{18}$. The concentration of the harvested protein P97 was measured using the Pierce BCA protein assay kit.

\section{Animals and vaccinations}

One dose $(2 \mathrm{~mL})$ of the multicomponent vaccine (test vaccine) contained $5 \times 10^{9}$ colonyforming units (CFU) for each inactivated App serotypes 1,2 , and $5 ; 50 \mu \mathrm{g}$ of the Apx toxoids from each App serotypes 1, 2, and 5; $2 \times 10^{8} \mathrm{CCU}$ of inactivated Mhp; and $25 \mu \mathrm{g}$ of the purified rP97. The vaccine was formulated at a ratio of $1: 1$ with IMS 1313 adjuvant.

Eighteen 3-week-old pigs of both sexes were used in this study. All pigs were negative for App antibodies (IDEXX APP-ApxIVAb Test, IDEXX Laboratories Inc., USA), and Mhp antibodies (Mycoplasma hyopneumoniae Antibody Test Kit, IDEXX Laboratories Inc., USA). The 18 pigs were randomly separated into three groups, as shown in Table 2. Pigs in group 1 were immunized intramuscularly with the test vaccine twice over a 3-week interval. Pigs in group 2 were vaccinated intramuscularly twice with a commercial vaccine as per the manufacturer's instructions. The commercial vaccine used in this study (Nisseiken Swine APM Inactivated Vaccine; Nisseiken Co. Ltd., Japan) contains $1 \times 10^{8}$ CFU inactivated Mhp, 5 x $10^{9} \mathrm{CFU}$ for each inactivated App serotype 1, 2, and 5 , and recombinant proteins ApxI, II, and III (80-120 $\mu \mathrm{g})$. Pigs in group 3 were injected with PBS formulated with adjuvant as a negative control group.

The experimental design is shown in Fig. 1. Blood samples were taken at $0,1,3,5$, and 6 weeks after the first immunization for examination of antibodies. At 2 weeks after the second vaccination, two pigs from each group were sacrificed and their lungs were subjected to examination for histopathology. The remaining pigs were challenged intratracheally as previously described $^{2,11}$ with $1 \times 10^{5}$ CFU of each wild-type App serotypes 1, 2, and 5 . All pigs were sacrificed at 7 days postchallenge, and their lungs were collected for isolation of the challenge Apps and determination of their lung lesion score, as described by Inzana et al. ${ }^{27}$. Briefly, a score of 0 indicates healthy lung; $1,<25 \%$ lung involvement with localized congestion only; 2, 25-50\% lung 
involvement with consolidation or congestion only and no necrosis or pleural lesions; 3, 51-75\% lung involvement with some consolidation, congestion, mild pleural lesions, and congestion; 4, 76-100\% lung involvement with pneumonia and consolidation, extensive pleural adhesions, and necrotic lesions.

\section{Isolation of App from challenged pigs}

To determine the presence of the challenge Apps, the cranial lobe and the portions of lung that exhibited lesions were seared superficially, incised, sampled with a sterile cotton swab, and then streaked on a BHI agar plate containing $10 \mu \mathrm{g} / \mathrm{mL}$ NAD. After $24 \mathrm{~h}$ of incubation at $37^{\circ} \mathrm{C}$ and $5 \% \mathrm{CO}_{2}$, the colonies were tested for NAD dependence and satellitism. The identity of suspected colonies was confirmed by nested polymerase chain reaction $(P C R)^{28}$. Briefly, $5 \mu \mathrm{L}$ of the suspended colony was mixed with the PCR premix containing $2 \times$ Master Mix (Enzynomics, Korea), and 10 pmol of the primers listed in Table 1. The first PCR was carried out using primers P1, P2, and the nested PCR was carried out using primers P3, P4. Thermal cycling conditions included initial denaturation at $95^{\circ} \mathrm{C}$ for $5 \mathrm{~min}$; followed by 35 cycles of $95^{\circ} \mathrm{C}$ for $1 \mathrm{~min}, 52^{\circ} \mathrm{C}$ for $1 \mathrm{~min}, 72^{\circ} \mathrm{C}$ for $1 \mathrm{~min}$; and a final extension at $72^{\circ} \mathrm{C}$ for $10 \mathrm{~min}^{28}$. Enzyme-linked immunosorbent assay (ELISA)

A Mhp antibody test kit (IDEXX Laboratories Inc., Westbrook, USA) was used to evaluate antibody production against Mhp in sera from the pigs. In addition, purified rP97 was coated on wells at $10 \mathrm{ng}$ per well to detect specific antibodies against rP97.

Table 1. Primers used in this study

\begin{tabular}{lllll}
\hline Primer & Sequence (5'-3') & Target & $\begin{array}{l}\text { PCR } \\
\text { product (bp) }\end{array}$ & Reference \\
\hline P1 & TGGCACTGACGGTGATGA & apxIVA & 442 & 28 \\
P2 & GGCCATCGACTCAACCAT & & & \\
P3 & GGGGACGTAACTCGGTGATT & apxIVA & 378 & \\
P4 & GCTCACCAACGTTTGCTCAT & & & \\
\hline
\end{tabular}

Table 2. Animal experimental design and reisolation of challenge Apps

\begin{tabular}{|c|c|c|c|c|}
\hline \multirow[t]{2}{*}{ Groups } & \multicolumn{3}{|c|}{ No. of pigs } & \multirow{2}{*}{$\begin{array}{l}\text { Reisolation of the Apps } \\
\text { after challenge }(\%)^{*}\end{array}$} \\
\hline & Total & Nonchallenge & Challenge & \\
\hline Test vaccine & 7 & 2 & 5 & $1 / 5(20 \%)$ \\
\hline Commercial vaccine & 4 & 2 & 2 & $2 / 2(100 \%)$ \\
\hline Negative control & 7 & 2 & 5 & $5 / 5(100 \%)$ \\
\hline
\end{tabular}

*Percentage of pigs in each group in which App could be detected.

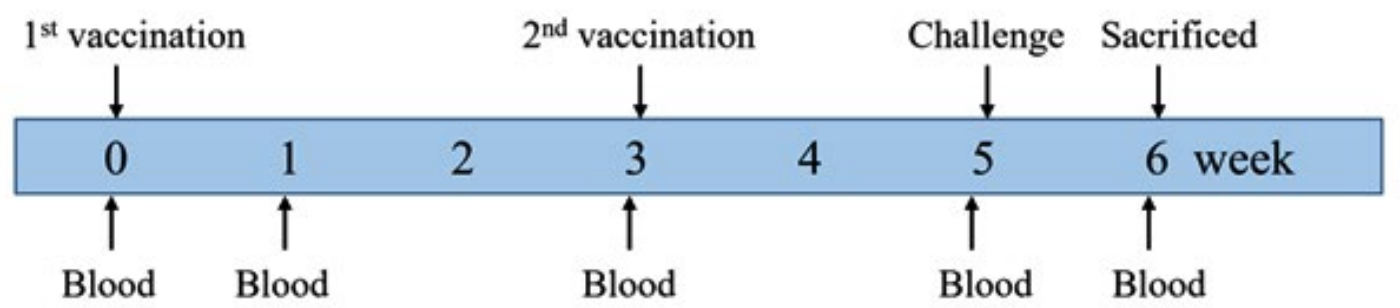

Fig. 1. Animal experimental design. Pigs were immunized with two doses of the test vaccine, the commercial vaccine, or PBS at 0 and 3 weeks, and challenged with virulent App strains at 5 weeks after the first vaccination. Blood samples were collected at $0,1,3,5$, and 6 weeks after the first vaccination. 
To evaluate the levels of antibodies against App, the 42-kDa outer membrane protein (OMP) was extracted ${ }^{29}$ and $100 \mathrm{ng}$ of the purified OMP was coated onto each well for ELISA. Plates coated with a mixture of $10 \mathrm{ng}$ of the purified Apx toxins from each App serotypes, were used to detect Apx-specific antibodies.

The antigens were incubated in 100 $\mu \mathrm{L}$ coating buffer overnight at $4^{\circ} \mathrm{C}$ using ELISA plates (Nunc, Denmark). After washing three times with $200 \mu \mathrm{L}$ of PBS containing $0.05 \%$ Tween 20 (PBST; Merck, USA) and blocking for $2 \mathrm{~h}$ with $1 \%$ bovine serum albumin (Merck, USA) in PBST, $1 / 1,000$ dilutions of individual sera were added and incubated for $2 \mathrm{~h}$ at $37^{\circ} \mathrm{C}$. This was followed by three washes, then the horseradish peroxidase-conjugated secondary antibodies $(1 / 5,000)$ (Goat anti-pig IgG H\&L; Abcam, UK) were incubated for $1 \mathrm{~h}$. Then the plates were washed with PBST, and incubated with 3,3',5,5'-tetramethylbenzidine substrate (Abcam, UK) for $3 \mathrm{~min}$ at room temperature. After adding $2 \mathrm{~N} \mathrm{H}_{2} \mathrm{SO}_{4}$, the absorbance was measured at 450 $\mathrm{nm}$ using an $\mathrm{EPOCH}$ reader (BioTek Instruments, Inc., USA).

\section{Statistical analysis}

Statistical analysis and preparation of graphs were performed using GraphPad Prism v6

A

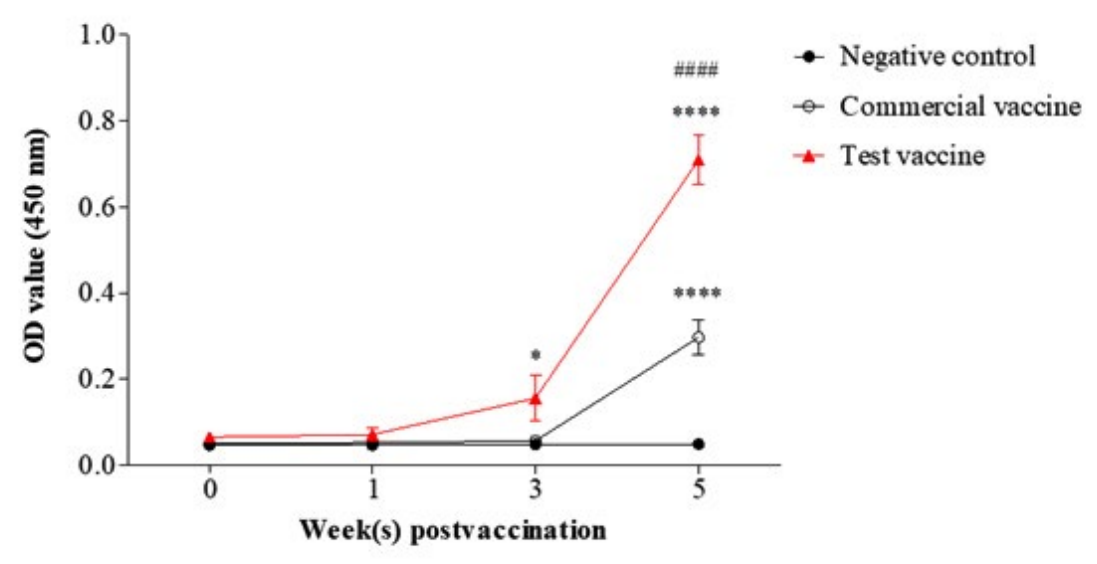

B

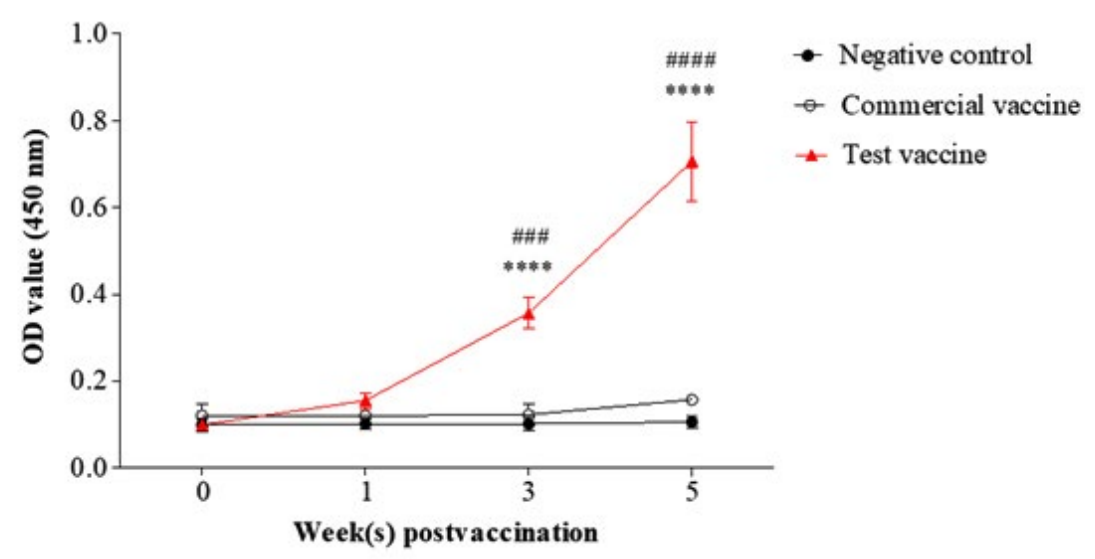

Fig. 2. Specific antibody responses against $\mathrm{Mhp}(\mathrm{A})$ and $\mathrm{rP97}(\mathrm{B})$ in the test vaccine $(\boldsymbol{\Delta})$, the commercial vaccine $(\mathrm{o})$, and the negative control groups $(\bullet)$. Data are presented as means \pm SD. The asterisks and hashes indicate significant differences $(* p<0.05 ; * * * * p<0.001$, compared with the negative control group. \#\#\#,\#\# $p<0.001$, compared with the commercial vaccine group). OD value $(450 \mathrm{~nm})$, absorbance at $450 \mathrm{~nm}$. 
software (GraphPad Software, USA). An analysis of variance (ANOVA) was used to evaluate the differences between experimental groups.

\section{RESULTS}

\section{Induction of antibodies against Mhp}

To evaluate the immunogenicity of the test vaccine against Mhp, the levels of antibodies against Mhp or P97 were determined using the commercial ELISA kit or an in-house P97-based indirect ELISA.

Analysis of the anti-Mhp IgG levels in the sera demonstrated that the pigs immunized with the test vaccine generated significantly higher antibody levels than the negative control group at 3 weeks $(p=0.014)$ and 5 weeks $(p<0.001)$ after the first vaccination (Fig. 2.A). Furthermore, the antibody titer against Mhp in the test vaccine group was 2.6 times higher than that in the commercial vaccine group, and this difference was significant $(p<0.001)$ (Fig. 2.A).

Significant levels of P97-specific antibodies $(p<0.001)$ were observed in the serum samples from the test vaccine group, while they were not detectable in the sera of pigs immunized with the commercial vaccine or in pigs in the negative control group at 3 and 5 weeks after the first vaccination (Fig. 2.B). These results illustrate that the test vaccine showed good ability to induce production of antibodies against Mhp.

Induction of antibodies against App

The $42 \mathrm{kDa}$ OMP is predominant and is conserved among App serotypes ${ }^{30,31}$. Therefore, App OMP was used as a coating antigen in ELISA

A

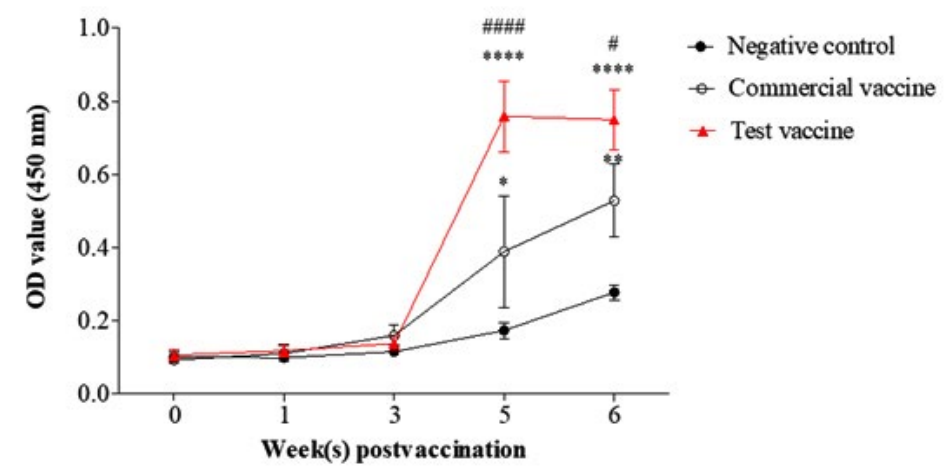

B

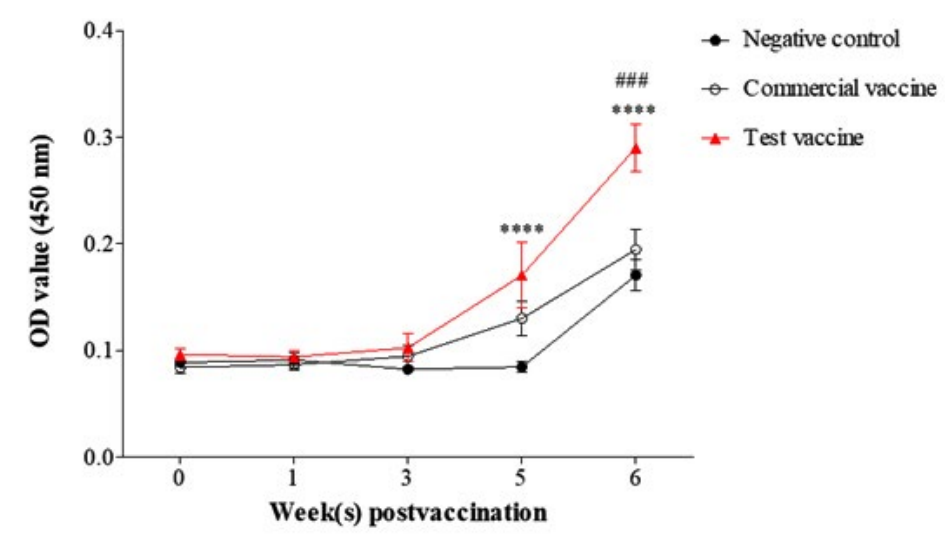

Fig. 3. Specific antibody responses against $42 \mathrm{kDa} O M P(A)$ and $A p x$ toxoids $(B)$ in the test vaccine $(\boldsymbol{\Delta})$, the commercial vaccine $(0)$, and the negative control groups $(\bullet)$. Data are presented as means \pm SD. The asterisks and hashes indicate significant differences $\left({ }^{*} p<0.05 ;{ }^{* *} p<0.01 ; * * * * p 0.001\right.$, compared with the negative control group. \#p<0.05; \#\#\#, \#\#\# p<0.001, compared with the commercial vaccine group). OD value (450 nm), absorbance at $450 \mathrm{~nm}$. 
to evaluate the antibody levels against App as a previous report ${ }^{32}$ and in the commercial kits (VDProAPP2 AB ELISA and VDProAPP5 AB ELISA; Median Diagnostics Inc., Korea). The levels of OMPspecific antibodies were increased dramatically in the sera of pigs vaccinated with the test vaccine and the commercial vaccine at 2 weeks after the second vaccination and remained at a high level at one week post challenge (Fig. 3.A). In addition, significantly higher levels of IgG against OMP were observed in the test vaccine group than in the commercial vaccine group at these time points ( $p<0.001$ and $p=0.018$, respectively) (Fig. 3.A).

The same pattern was seen for the levels of IgG against $A p x$ toxins in the test vaccine group (Fig. 3.B), which were significantly higher than those in the commercial vaccine and negative control groups $(p<0.001)$. In contrast, there was no significant difference between the antibody levels in the commercial vaccine and negative control groups. These results demonstrated that the test vaccine could induce better production of antibodies against App and Apx toxins than the commercial vaccine.

\section{Protective efficacy of vaccine against App challenge}

To evaluate the protection against virulent Apps provided by the test vaccine, pigs in all three groups were challenged with virulent App serotypes 1, 2, and 5. The lung lesion scores of the challenged pigs are shown in Fig. 4 and 5. Nonchallenged pigs in all groups showed no lung lesions. The test vaccine group showed lower lung lesion scores than the commercial vaccine and negative control groups with mean scores of $0.6 \pm 0.5,1.5 \pm 0.7$, and $2.4 \pm 0.5$, respectively. A significant difference in lung lesion scores was observed between the test vaccine group and the negative control group ( $p=0.002$ ). No significant difference in lung lesion scores was detected between the commercial vaccine and negative control groups $(p=0.196)$.

The protection provided by the vaccines was also assessed by the recovery of App from challenged pigs as shown in Table 2 . The recovery rates from the lungs of pigs in the test vaccine, the commercial vaccine, and the negative control groups challenged with Apps were $20 \%(1 / 5)$, $100 \%(2 / 2)$, and $100 \%(5 / 5)$, respectively.

\section{DISCUSSION}

Coinfection with Mhp and App is very frequent in swine farms ${ }^{13}$. These diseases cause more severe lesions in pigs than single infections and lead to major economic losses in the swine industry ${ }^{13}$. Therefore, there is a strong demand for the development of multivalent vaccines against Mhp and App, because these reduce the number of vaccinations and hence reduce the high labor costs of the vaccinations and the level of injectioninduced stress in pigs. To address this demand, in this study, we developed a promising multidisease vaccine and compared the immune responses and the protective efficacy it induced against Mhp and App infections in pigs with those induced by a commercial vaccine. To our knowledge, this is the first reported development of a multivalent vaccine comprising a new formulation of Mhp bacterin, P97, App serotypes 1, 2, 5, and ApxI, II, and III toxoids.

A previous report has demonstrated that several of the OMPs from App, which are present in App bacterins, are conserved across all serotype $^{33}$. A vaccine containing a combination of these proteins and Apx toxoids can protect challenged pigs against lung lesion formation

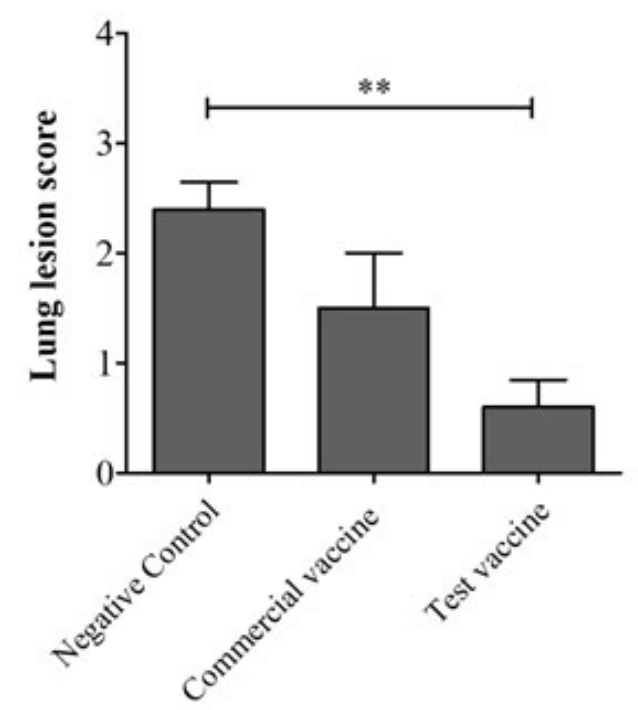

Fig. 4. Lung lesion scores of pigs vaccinated with the test vaccine, the commercial vaccine, or PBS after challenge with virulent App strains. Data are presented as means \pm SD. The asterisks indicate significant differences. $* *$ $p<0.01$. 
better than one containing only Apx toxoids ${ }^{34}$. Wu et al. (2018) showed that a vaccine containing App bacterins significantly reduced gross lesion scores compared with those observed after a vaccine without App bacterins ${ }^{4}$. These results indicated that App bacterins contributed to reducing lung lesions.

The test vaccine developed in the present study effectively stimulated the production of antibodies against $42 \mathrm{kDa}$ OMP and Apx toxins. The antibody levels were significantly higher than those induced by the commercial vaccine and in the negative control group at 5 weeks after the first vaccination and at one week post challenge (Fig. 3.A and B). This may be because in the test vaccine, the Apx toxoids were extracted directly from cultures of App, while the Apx proteins in the commercial vaccine were expressed in and purified from $E$. coli. The immune responses and protective efficacy induced by the recombinant Apx toxins and the inactivated native Apx toxins were also studied. Mei et al. (2006) reported that higher antibody levels were induced by native ApxI toxoids than by recombinant full-length $\mathrm{ApxIA}^{3}$. Yan et al. (2007) also reported higher antibody titers and a higher protection rate in mice injected with
A

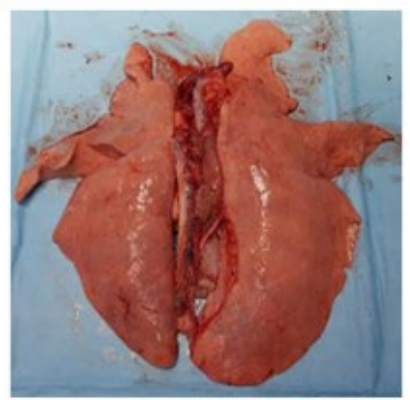

$\mathrm{C}$

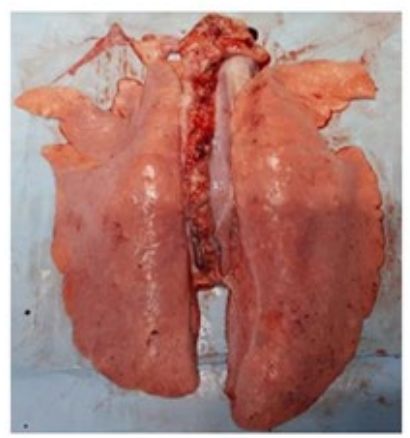

$\mathrm{E}$

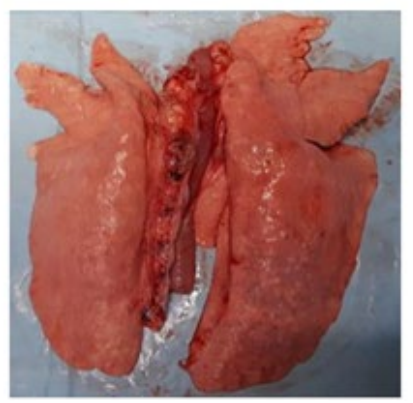

$\mathrm{B}$

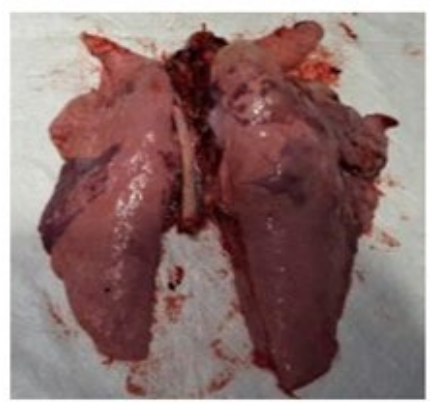

$\mathrm{D}$

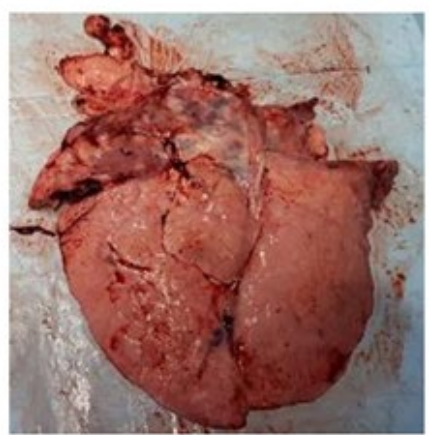

$\mathrm{F}$

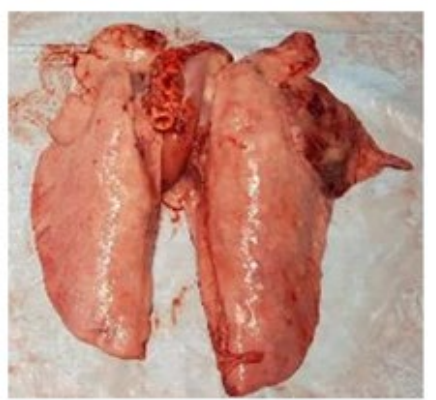

Fig. 5. Gross lung lesions of pigs after challenge with virulent App strains. Lungs of pigs in the negative control group, nonchallenged $(A)$ and challenged $(B)$; lungs of pigs in the commercial vaccine group, nonchallenged $(C)$ and challenged (D); lungs of pigs in the test vaccine group, nonchallenged $(E)$ and challenged $(F)$. 
native Apxll compared with recombinant ApxIIA ${ }^{35}$. In our study, we observed significantly higher levels of antibodies against Apx toxins in pigs immunized with the test vaccine than in those immunized with the commercial vaccine. Our results are consistent with those of previous studies in which native Apx toxoids induced a better immune response and protection than recombinant Apx toxins.

The formulation, induction of immune responses, and protective efficacy of the Mhp vaccine were evaluated and reported in our previous work ${ }^{36}$. The Mhp vaccine completely protected vaccinated pigs against Mhp challenge. Thus, a challenge experiment with Mhp was not conducted in this study. In this study, the antibody levels against Mhp and rP97 produced in the test vaccine pigs were significantly higher than those in the other groups at 3 and 5 weeks after the first vaccination (Fig. 2.A and B). These results suggest that the test vaccine could effectively induce the production of antibodies against both App and Mhp.

The protective efficacy of the test vaccine was evaluated by challenge with virulent App. The test vaccine provided better protection for challenged pigs as assessed by lung lesion scores (Fig. 4 and 5) and the rate of recovery of the challenge App from the lung tissues of pigs in each group (Table 2). The higher specific antibody levels in the test vaccine-immunized pigs may have contributed to the lower lung lesion scores in this group. The highest rates of recovery of the challenge App were recorded in the negative control group and the commercial vaccine group. This result implied that the test vaccine could more effectively protect pigs and eliminate the challenge App bacteria than the commercial vaccine. Further studies are required to assess the protective efficacy of this vaccine against challenge with highly virulent Mhp.

In conclusion, this multicomponent vaccine induced relatively high levels of antibodies against both App and Mhp, and could effectively protect pigs against App challenge, indicating that it could be a candidate vaccine for prevention of these two economically important respiratory diseases.

\section{ACKNOWLEDGMENTS}

\section{None.}

\section{CONFLICT OF INTEREST}

The authors declare that there is no conflict of interest.

\section{AUTHORS' CONTRIBUTION}

All authors listed have made a substantial, direct and intellectual contribution to the work, and approved it for publication.

\section{FUNDING}

This work was supported by the Technology Development Program to Innovac Co. S2540568, and funded by the Ministry of SMEs and Startups (MSS, Korea). This study was supported by 2017 Research Grant from Kangwon National University(No. 520170388).

\section{DATA AVAILABILITY}

All datasets generated during this study are included in the manuscript.

\section{ETHICS STATEMENT}

All animal experiments were performed at the laboratory animal facility and conducted with the approval of the Institutional Animal Care and Use Committee (Approval number: KW180611-2).

\section{REFERENCES}

1. Sassu EL, Bosse JT, Tobias TJ, Gottschalk M, Langford PR, Hennig-Pauka I. Update on Actinobacillus pleuropneumoniae-knowledge, gaps and challenges. Transbound Emerg Dis. 2018;65(Suppl 1):72-90. doi: 10.1111/tbed.12739

2. Liu J, Chen X, Lin L, et al. Potential use an Actinobacillus pleuropneumoniae double mutant strain $\triangle a p x I / C \Delta a p x I V A$ as live vaccine that allows serological differentiation between vaccinated and infected animals. Vaccine. 2007;25(44):7696-7705. doi: 10.1016/j.vaccine.2007.07.053

3. Mei L, Zhou R, Lu HS, et al. Study on the immunogenicity of $\mathrm{N}$-terminal polypeptide of RTX toxin I of Actinobacillus pleuropneumoniae. Chin J Biotechnol. 2006;22(1):39-45. doi: 10.1016/S18722075(06)60005-8

4. Wu HC, Yeh PH, Hsueh KJ, Yang WJ, Chu CY. Recombinant ApxIV protein enhances protective efficacy against Actinobacillus pleuropneumoniae in mice and pigs. J Appl Microbiol. 2018;124(6):13661376. doi: 10.1111/jam.13726

5. Ramjeet M, Deslandes V, Goure J, Jacques M. Actinobacillus pleuropneumoniae vaccines: from bacterins to new insights into vaccination strategies. Anim Health Res Rev. 2008;9(1):25-45. doi: 10.1017/ S1466252307001338 
6. Frey J. Biological safety concepts of genetically modified live bacterial vaccines. Vaccine. 2007;25(30):55985605. doi: 10.1016/j.vaccine.2006.11.058

7. Detmer A, Glenting J. Live bacterial vaccines-a review and identification of potential hazards. Microb Cell Fact. 2006;5:23. doi: 10.1186/1475-2859-5-23

8. Bosse JT, Li Y, Sarkozi R, et al. Proposal of serovars 17 and 18 of Actinobacillus pleuropneumoniae based on serological and genotypic analysis. Vet Microbiol. 2018;217:1-6. doi: 10.1016/j.vetmic.2018.02.019

9. Frey J. Virulence in Actinobacillus pleuropneumoniae and RTX toxins. Trends Microbiol. 1995;3(7):257-261. doi: 10.1016/S0966-842X(00)88939-8

10. Komal JP, Mittal KR. Grouping of Actinobacillus pleuropneumoniae strains of serotypes 1 through 12 on the basis of their virulence in mice. Vet Microbiol. 1990;25(2-3):229-240. doi: 10.1016/03781135(90)90080-F

11. Yuan F, Liu J, Guo Y, et al. Influences of ORF1 on the virulence and immunogenicity of Actinobacillus pleuropneumoniae. Curr Microbiol. 2011;63(6):574580. doi: 10.1007/s00284-011-0016-0

12. Lee KE, Choi HW, Kim HH, Song JY, Yang DK. Prevalence and characterization of Actinobacillus pleuropneumoniae isolated from Korean pigs. J Bacteriol Virol. 2015;45(1):19-25. doi: 10.4167/ jbv.2015.45.1.19

13. Maes D, Sibila M, Kuhnert P, Segales J, Haesebrouck F, Pieters M. Update on Mycoplasma hyopneumoniae infections in pigs: Knowledge gaps for improved disease control. Transbound Emerg Dis. 2018;65(Suppl 1):110-124. doi: $10.1111 /$ tbed.12677

14. Maes D, Segales J, Meyns T, Sibila M, Pieters M, Haesebrouck F. Control of Mycoplasma hyopneumoniae infections in pigs. Vet Microbiol. 2008;126(4):297-309. doi: 10.1016/j.vetmic.2007.09.008

15. Haesebrouck F, Pasmans F, Chiers K, Maes D, Ducatelle $R$, Decostere A. Efficacy of vaccines against bacterial diseases in swine: what can we expect? Vet Microbiol. 2004; 100(3-4):255-268. doi: 10.1016/j. vetmic.2004.03.002

16. Wilton JL, Scarman AL, Walker MJ, Djordjevic SP. Reiterated repeat region variability in the ciliary adhesin gene of Mycoplasma hyopneumoniae. Microbiology. 1998;144(Pt 7):1931-1943. doi: 10.1099/00221287-144-7-1931

17. Hsu T, Artiushin S, Minion FC. Cloning and functional analysis of the P97 swine cilium adhesin gene of Mycoplasma hyopneumoniae. J Bacteriol. 1997;179(4):1317-1323. doi: 10.1128/JB.179.4.13171323.1997

18. Barate AK, Cho Y, Truong QL, Hahn TW. Immunogenicity of IMS 1113 plus soluble subunit and chimeric proteins containing Mycoplasma hyopneumoniae P97 C-terminal repeat regions. FEMS Microbiol Lett. 2014;352(2):213-220. doi: 10.1111/1574-6968.12389

19. Marchioro SB, Sacristan Rdel P, Michiels A, et al. Immune responses of a chimaeric protein vaccine containing Mycoplasma hyopneumoniae antigens and LTB against experimental $M$. hyopneumoniae infection in pigs. Vaccine. 2014;32(36):4689-4694. doi: 10.1016/j.vaccine.2014.05.072
20. Lee SH, Lee S, Chae C, Ryu DY. A recombinant chimera comprising the R1 and R2 repeat regions of $M$. hyopneumoniae $\mathrm{P} 97$ and the $\mathrm{N}$-terminal region of $A$. pleuropneumoniae Apxlll elicits immune responses. BMC Vet Res. 2014;10:43. doi: 10.1186/1746-6148$10-43$

21. Yagihashi T, Nunoya T, Mitui T, Tajima M. Effect of Mycoplasma hyopneumoniae infection on the development of Haemophilus pleuropneumoniae pneumonia in pigs. Nihon Juigaku Zasshi. 1984;46(5):705-713. doi: 10.1292/jvms1939.46.705

22. Marois C, Gottschalk M, Morvan H, Fablet C, Madec $\mathrm{F}$, Kobisch M. Experimental infection of SPF pigs with Actinobacillus pleuropneumoniae serotype 9 alone or in association with Mycoplasma hyopneumoniae. Vet Microbiol. 2009;135(3-4):283-291. doi: 10.1016/j. vetmic.2008.09.061

23. Matthijs AMF, Auray G, Jakob V, et al. Systems immunology characterization of novel vaccine formulations for Mycoplasma hyopneumoniae bacterins. Front Immunol. 2019;10:1087. doi: 10.3389/ fimmu.2019.01087

24. Opriessnig T, Hemann M, Johnson JK, et al. Evaluation of diagnostic assays for the serological detection of Actinobacillus pleuropneumoniae on samples of known or unknown exposure. J Vet Diagn Invest. 2013;25(1):61-71. doi: 10.1177/1040638712469607

25. Dao HT, Do VT, Truong QL, Hahn TW. Enhancement of Apx toxin production in Actinobacillus pleuropneumoniae serotypes 1,2 , and 5 by optimizing culture conditions. J Microbiol Biotechnol. 2020;30(7):1037-1043. doi: 10.4014/jmb.1912.12042

26. Hu X, Yan H, Liu K, et al. Identification and characterization of a novel stress-responsive outer membrane protein Lip40 from Actinobacillus pleuropneumoniae. BMC Biotechnol. 2015;15:106. doi: 10.1186/s12896-015-0199-8

27. Inzana TJ, Todd J, Veit HP. Safety, stability, and efficacy of noncapsulated mutants of Actinobacillus pleuropneumoniae for use in live vaccines. Infect Immun. 1993;61(5):1682-1686. doi: 10.1128/ IAI.61.5.1682-1686.1993

28. Schaller A, Djordjevic SP, Eamens GJ, et al. Identification and detection of Actinobacillus pleuropneumoniae by PCR based on the gene apxIVA. Vet Microbiol. 2001;79(1):47-62. doi: 10.1016/S03781135(00)00345-X

29. Van Den BJF. Actinobacillus pleuropneumoniae subunit vaccine. United States Patent 5648081. 1997.

30. Haesebrouck F, Chiers K, Van Overbeke I, Ducatelle R. Actinobacillus pleuropneumoniae infections in pigs: the role of virulence factors in pathogenesis and protection. Vet Microbiol. 1997;58(2-4):239-249. doi: 10.1016/S0378-1135(97)00162-4

31. Maclnnes JI, Rosendal S. Analysis of major antigens of Haemophilus (Actinobacillus) pleuropneumoniae and related organisms. Infect Immun. 1987;55(7):16261634. doi: 10.1128/IAI.55.7.1626-1634.1987

32. Eamens GJ, Gonsalves JR, Whittington AM, Turner B. Evaluation of serovar-independent ELISA antigens of Actinobacillus pleuropneumoniae in pigs following vaccination or experimental challenge with respiratory 
pathogens and natural $A$. pleuropneumoniae serovar 1 challenge. Aust Vet J. 2012;90(12):490-498. doi: 10.1111/j.1751-0813.2012.01008.x

33. Rapp VJ, Ross RF. Antibody response of swine to outer membrane components of Haemophilus pleuropneumoniae during infection. Infect Immun. 1986;54(3):751-760. doi: 10.1128/IAI.54.3.751760.1986

34. Madsen ME, Carnahan KG, Thwaits RN. Evaluation of pig lungs following an experimental challenge with Actinobacillus pleuropneumoniae serotype 1 and 5 in pigs inoculated with either hemolysin protein and/ or outer membrane proteins. FEMS Microbiol Lett. 1995;131(3):329-335. doi: 10.1111/j.1574-6968.1995. tb07796.x

35. Yan KX, Liu JJ, Wu B, et al. Cloning, expression and immunogenic characterization of the apxIIA gene of Actinobacillus pleuropneumoniae. Chin J Agric Biotechnol. 2007;4(1):63-67. doi: 10.1017/ S1479236207001222

36. Hahn TW, Barate KA, Kim KJ, Shin WS. Vaccine composition comprising recombinant protein for preventing swine Mycoplasma infection. United States Patent 20190177375; 2019. 